Cinémas

Revue d'études cinématographiques

Journal of Film Studies

\title{
Du film, du texte et du rêve, en tant qu'objets et activités symboliques
}

\section{Marc-F. Gélinas}

Volume 2, numéro 1, automne 1991

Le Scénario

URI : https://id.erudit.org/iderudit/1001054ar

DOI : https://doi.org/10.7202/1001054ar

Aller au sommaire du numéro

Éditeur(s)

Cinémas

ISSN

1181-6945 (imprimé)

1705-6500 (numérique)

Découvrir la revue

Citer cet article

Gélinas, M.-F. (1991). Du film, du texte et du rêve, en tant qu'objets et activités symboliques. Cinémas, 2(1), 121-135. https://doi.org/10.7202/1001054ar

\section{Résumé de l'article}

Bien que le rêve soit un objet symbolique et spontané par excellence (rêve et rêveur sont liés) et que le film et le texte soient des objets symboliques à enclenchement volontaire (film, texte, spectateur et lecteur sont séparés), au niveau de leur présentation, film, texte et rêve partagent des caractéristiques semblables. Le présent article cherche à préciser les diverses activités symboliques qui entrent en jeu dans le rêve, le texte et le film et à mettre en valeur les points de jonction qui font que le rêve, le texte et le film partagent un terrain commun qui nous éclaire sur la forme que prend le scénario et sur sa relation au film. 


\title{
Du film, du texte et du rêve, en tant qu'objets et activités symboliques
}

\section{Marc-F. Gélịnas}

\begin{abstract}
RÉSUMÉ
Bien que le rêve soit un objet symbolique et spontané par excellence (rêve et rêveur sont liés) et que le film et le texte soient des objets symboliques à enclenchement volontaire (film, texte, spectateur et lecteur sont séparés), au niveau de leur présentation, film, texte et rêve partagent des caractéristiques semblables. Le présent article cherche à préciser les diverses activités symboliques qui entrent en jeu dans le rêve, le texte et le film et à mettre en valeur les points de jonction qui font que le rêve, le texte et le film partagent un terrain commun qui nous éclaire sur la forme que prend le scénario et sur sa relation au film.
\end{abstract}

\begin{abstract}
Although the dream is above all a spontaneous symbolic object (dream and dreamer are linked) and the film and the text are symbolic objects under voluntary control (film, text, spectator and reader are separate), with respect to their presentation, film, text and dream share similar characteristics. This article seeks to specify the various symbolic activities that come into play in dreams, texts and films and to highlight the points of connection where dream, text and film share common ground, shedding light on the form the screenplay takes and on its relationship to the film.
\end{abstract}

Cette réflexion est celle d'un créateur praticien de la scénarisation et de son enseignement. Elle vient d'une insatisfaction face au dogmatisme de plusieurs textes sur la pratique de la scénarisation et de ce qui nous semble être leur manque de vision. 
Elle prend aussi sa source dans cette distance que nous avons perçue entre ce que nous appellerons d'une part nos manœuvres de professeur, nos discours sur l'œuvre et sur l'acte d'écrire, et d'autre part la réalité beaucoup plus complexe et sentie de ces choses.

La présente réflexion est un constat sur la nature probable du film, du texte et du scénario. Elle dégage certaines nécessités du film et du texte ainsi que l'impact de ces nécessités sur celles du scénario. Elle n'aborde pas l'acte d'écrire lui-même qui demeure mystérieux.

Nous entendons par film, ici, tout ce qui produit un effet cinématographique, images en mouvement et son, couvrant l'ensemble des supports, des genres et des œuvres audiovisuels. Nous les abordons comme s'ils ne faisaient qu'un, puisque nous croyons que le poids des caractéristiques communes entre eux est beaucoup plus grand que celui de leurs différences. Ceci dit, en sachant cependant qu'il y a dans ces différences matière à une vaste et passionnante réflexion encore à faire. Nous entendons par texte, ici, tout document écrit et en particulier le scénario auquel nous allons nous intéresser de plus près.

\section{La réalité fondamentale du film}

Imaginons que nous avons de la lumière, n'importe quelle lumière, projetée sur un écran et du son, n'importe quel son. Avons-nous un film? Pour qu'il y ait film, la lumière doit être image et le son doit être son, c'est-à-dire autre chose que du bruit. Et comment passe-t-on de la lumière à l'image et du bruit au son? Cette magie s'opérerait, entre autres, à travers l'intention projetée et perçue.

À la limite, donc, celui qui déciderait de projeter une certaine lumière et de faire entendre un certain son selon une organisation et une séquence données, aurait fait un film. Mais à moins que ce son et cette lumière ne soient perçus par d'autres comme le résultat d'une intention et qu'ils ne soient porteurs d'un certain sens, cet individu n'aurait fait œuvre que pour lui-même, ce qui équivaudrait presque à n'avoir rien fait. L'objet seul, ce que nous appelons habituellement l'œuvre, la matérialisation de l'œuvre, nous apparaît donc comme une condition nécessaire mais insuffisante à son existence en tant qu'œuvre. Tout comme l'intention énoncée ou perçue seule. De plus, si le seul sens qu'on puisse tirer d'un certain son et d'une certaine lumière projetée sur un écran est qu'il y a là une intention de faire une œuvre, on n'est en effet pas beaucoup plus avancé.

$\mathrm{Si}$, dans notre exploration du film, on en reste au niveau de la lumière et du son, on se bute donc rapidement au même cul-de- 
sac qu'en restant au niveau de l'intention: l'absence de sens. Pour avancer il faut passer ailleurs, par le spectateur, et revenir ensuite à la matérialisation de l'œuvre. Les œuvres sont des créations de l'esprit pour l'esprit, et elles sont également matérielles. C'est dans l'examen de cette dualité et dans le pont entre ces deux pôles que nous allons chercher des réponses; en examinant ce qu'il y a à l'écran, ce qu'il y a dans la salle, et le pont entre les deux.

Commençons par examiner ce qu'il y a à l'écran par le biais du spectre des œuvres qui s'étend du documentaire à une extrémité, à la fiction à l'autre. Partons des définitions communément admises (Le Petit Robert):

Documentaire: film instructif destiné à montrer des documents, des faits enregistrés et non élaborés pour l'occasion (opposé à film de fiction).

Fiction: mensonge. Fait imaginé (opposé à réalité). Création de l'imagination, en littérature.

Quelles seraient les caractéristiques des films qui répondraient le plus à chacune de ces deux définitions? En d'autres mots, que seraient le documentaire et la fiction absolus? D'après la définition, l'objet du documentaire absolu serait d'informer de façon absolue sur un sujet donné. Son idéal serait la transparence totale, arriver à présenter la chose elle-même, arriver à livrer au spectateur le sujet, le réel décrit lui-même. Le documentaire absolu tirerait tous ses effets des caractéristiques objectives de son sujet; il serait construit tout en dénotation de son sujet et ne référerait qu'à lui. Il serait entièrement au service de son sujet.

À l'autre extrémité du spectre des œuvres, la fiction absolue aurait pour objet de présenter une chose absolument imaginée. Son idéal serait de ne jamais référer à quoi que ce soit d'extérieur ou d'autre qu'elle-même, puisque toute référence à une chose existante irait contre la définition. La fiction absolue tirerait son effet de ses rythmes, de ses motifs, de sa façon d'établir ses conventions à l'intérieur de l'œuvre et de jouer avec elles. Elle serait construite selon un système de dénotation et de connotation clos, refermé sur lui-même. La fiction absolue serait entièrement à son propre service.

Aux deux extrémités du spectre nous aurions donc deux œuvres:

— le documentaire absolu, entièrement au service de son sujet et

— la fiction absolue, entièrement à son propre service.

La première œuvre s'effacerait devant l'objet représenté pour aller jusqu'à le présenter à l'état brut et dans sa totalité. Le do- 
cumentaire absolu ne serait idéalement plus représentation mais présentation, il serait idéalement et absurdement la chose ellemême. La seconde œuvre rechercherait la création pure, totale; la coupure de toute référence à autre chose qu'elle-même. On sent bien que ni l'une ni l'autre de ces œuvres n'ont de sens. Que toutes les œuvres réelles se retrouvent entre ces deux extrêmes.

Paradoxalement donc, en poussant vers les extrémités opposées du spectre des œuvres, on arrive à la même conclusion: chacun des films ne pourrait avoir que lui-même comme sujet. Cela nous porte à croire que le documentaire absolu et la fiction absolue seraient probablement une seule et même chose qu'on serait tenté d'appeler le film absolu: de la lumière et du son sans autre signification que celle d'être un agencement de lumière et de son, un film...

Il découle de cet examen du documentaire absolu qu'il n'y aurait pas, dans la pratique, de film sans représentation. Et cette obligation de représentation signifierait qu'une des réalités fondamentales, sinon la réalité fondamentale du film, serait d'être un objet symbolique; que de regarder un film c'est s'engager dans une activité symbolique. La deuxième conclusion que nous tirons de cette discussion nous vient de notre examen de la fiction absolue et c'est la suivante: ce qui peut apparaître de prime abord comme de l'intrinsèquement cinématographique (la grammaire, la syntaxe, etc.) n'aurait pas de sens en soi. Sans la représentation, il n'y aurait pas de film. La grammaire s'exercerait à travers la représentation. On serait presque tenté d'affirmer qu'elle en fait partie intégrante. En tant que convention, elle en fait certainement partie.

Nous avons abordé cette partie de notre réflexion en faisant comme si documentaire et fiction pouvaient être des qualités intrinsèques de la lumière et du son à l'écran. Nous nous rendons maintenant compte qu'il n'en est rien. Les catégories d'œuvres ne sont pas à l'écran mais dans notre tête. Ce sont des grilles de lecture de l'intention et du sens des œuvres. Elles font plus partie du mode d'emploi du film que de l'objet lui-même.

Nous arrivons donc à la réponse à notre question: la réalité fondamentale du film serait d'être d'abord un objet symbolique. Regarder un film serait une activité symbolique. Au-delà de cela, l'intrinsèquement cinématographique n'existerait pour ainsi dire pas, sinon comme une pratique un peu spécifique de la représentation. 


\section{Film, texte et rêve se ressemblent}

Arrêtons-nous à la définition communément admise du symbole:

Être, objet ou fait qui, par sa forme ou sa nature, évoque spontanément (dans une société ou une civilisation donnée) quelque chose d'abstrait ou d'absent (Le Petit Robert).

Les objets des films n'y sont pas physiquement présents; il s'agit très évidemment à l'écran de symboles au sens premier de la définition. La fréquentation de ces symboles - regarder un film - est une activité symbolique. Cette activité symbolique est fabriquée et à fréquentation volontaire.

Avant même d'avoir formulé ces choses, nous utilisions dans nos cours une définition de l'œuvre audiovisuelle qui comprenait la phrase: «Le film se déroule hors de la volonté du spectateur, comme un rêve... " Phrase que nous avons répétée pendant un an avant de nous rendre compte que nous ne nous étions jamais vraiment arrêté à réfléchir à ce que cela pouvait bien vouloir signifier. Lorsque nous l'avons fait, nous nous sommes demandé: «Et si le film était un rêve?» Cette question fut très bientôt suivie de: «Et si le film était un rêve, que serait le scénario?» Pour amorcer l'exploration de ces questions, nous nous sommes d'abord donné des définitions élémentaires des principaux objets touchés.

Rêve: suite de phénomènes psychiques se produisant pendant le sommeil.

Rêverie: activité mentale qui n'est pas dirigée par l'attention et qui se soumet à des causes subjectives et affectives.

Film: agencement de lumière et de son produisant un effet donné.

Scénario: texte qui décrit le film de façon suffisante à son démarchage et à sa production.

Texte: agencement de mots écrits produisant un effet donné.

Mot: chacun des groupes de lettres correspondant à un sens isolable spontanément, dans le langage.

En nous penchant sur ces définitions, nous nous sommes vite rendu compte que la caractéristique fondamentale du texte est d'être, tout comme le film, un objet symbolique fabriqué et à fréquentation volontaire, tandis que celle du rêve est d'être l'activité symbolique naturelle et à fréquentation spontanée par excellence. Leur nature symbolique semble être l'assise même de ces trois activités. Nous allons en explorer les grandes caractéristiques. 
Déroulement hors du contrôle de ceux qui s'y adonnent

Le rêve, avec son contenu, son point de vue, son rythme, l'ensemble de son déroulement, surgit spontanément chez le rêveur et se déroule hors de son contrôle. Le film et le texte ne surgissent pas spontanément chez le spectateur et le lecteur. Ils nécessitent plutôt une mise en disponibilité volontaire de leur part. Une fois la disponibilité du spectateur acquise, le film lui impose son contenu, son point de vue, son rythme, l'ensemble de son déroulement, hors de son contrôle comme le rêve. Le texte, pour sa part, nécessite (relativement parlant) la participation active du lecteur plutôt que sa simple mise en disponibilité. Une fois cette participation acquise, le texte, tout comme le rêve et le film, impose son contenu, son point de vue, son rythme et l'ensemble de son déroulement hors du contrôle du lecteur.

Mais le texte opère selon un niveau de définition très différent de celui du film. Le film nous donne un personnage, par exemple, avec un gabarit, un visage, une voix, des gestes et nous sommes prisonniers de ce qu'il nous donne. Le texte, par contre, décrit ou évoque le personnage et chacun l'imagine à sa guise. Là où nous dirons que le film est imposé, nous dirons que le texte est balisé. Par balisé, nous entendons que la trajectoire, le cheminement du lecteur à travers le texte passe par des repères imposés. À partir de ces repères, le lecteur a non seulement la liberté, mais aussi l'obligation d'établir sa propre représentation de ce qui est dit. Le lecteur imagine sa propre vision du paysage, mais ne contrôle pas l'itinéraire du voyage à travers le texte.

Nous avons donc trois activités symboliques qui se déroulent à des degrés divers hors du contrôle de celui qui s'y adonne:

- une activité naturelle, spontanée, totalement envahissante, le rêve;

— une fabriquée, nécessitant une mise en disponibilité préalable, et où presque tout est imposé, le film;

- une autre, fabriquée et exigeant une parícipation active à la fois pour ce qui est de son déroulement physique et de son interprétation, le texte.

Après leur nature symbolique, le déroulement hors du contrôle de celui qui s'y adonne nous semble être la caractéristique commune la plus importante entre le film, le texte et le rêve.

\section{Objets trompe-sens et trompe-gravité}

Le film, le texte et le rêve sont des objets trompe-sens. Le rêve crée spontanément dans notre tête quelque chose de neuf à 
partir de bribes ressemblant à des perceptions directes du monde extérieur par tous nos sens, mais sans que ceux-ci ne soient réellement sollicités: il recrée autant des touchers, des sons, des goûts, des odeurs que des images. Cela donne un assemblage parfois surréaliste - d'éléments surtout figuratifs.

Le film, lui, ne fait pas semblant de passer par les sens comme le rêve; il transite réellement par l'oreille et l'œil: l'oreille entend des vrais sons et l'œil perçoit de la vraie lumière. Le registre du film est cependant plus étroit que celui du rêve; il est amputé du goût, de l'odorat et du toucher. Par contre, le film jouit d'une grande virtuosité sonore et visuelle qui lui donne la capacité de créer et d'agencer des représentations d'éléments pleinement caractérisées et facilement identifiables, comme le rêve; des éléments qui de ce fait possèdent tout ce qu'il faut pour devenir des porteurs de sens, c'est-à-dire des symboles à l'intérieur de son déroulement. À la limite, cette virtuosité visuelle et sonore du film, la perfection de ses représentations, débouche sur l'effet de réalité qui est une manifestation trompesens de la vue et de l'ouïe. Il y a cependant une autre manifestation qui nous semble plus déterminante que l'effet de réalité: il s'agit du fait que la caméra et le micro deviennent les yeux et les oreilles du spectateur, et surtout que le spectateur est ainsi libéré des contraintes de son corps et de la gravité; qu'il a toute liberté pour se déplacer dans l'espace. Le film opère donc à travers des perceptions réelles mais qui se présentent au spectateur en trompe-sens et en trompe-gravité exactement comme s'il s'agissait d'un rêve.

$\mathrm{Vu}$ au premier degré, le texte ne partage pas les caractéristiques trompe-sens du film et du rêve. En effet, la lecture du texte (du scénario) ne sollicite qu'un seul sens, la vue et pas du tout de la même façon que le cinéma. Le texte ne nourrit la vue que de symboles graphiques qui représentent des mots. Ces mots sont eux-mêmes des symboles de choses qu'on peut percevoir pour beaucoup d'entre elles - directement par les sens; les mots, le langage sont déjà une première médiatisation, une première représentation et le texte est une représentation de cette première représentation. On est déjà, avec le texte, au deuxième niveau d'abstraction.

De plus, le texte fait passer par l'œil sous forme de signes abstraits (l'écriture) ce qui normalement devrait transiter de façon concrète par l'ouïe (le langage). Il y a donc non seulement double abstraction mais aussi substitution du sens (de l'organe) qui perçoit; l'œil remplace l'oreille. De plus, souvent, le langage représente des choses qu'on percevrait directement par la vue. Avec le texte il y a donc non seulement double abstraction mais 
aussi souvent double substitution: on passe de l'œil pour la perception brute des choses, à l'oreille pour une représentation par le langage de ces choses, à l'œil de nouveau pour la représentation recodifiée par l'écriture.

La fréquentation du texte tient souvent lieu de perceptions brutes de choses par les sens et nous cherchons alors spontanément à reconstituer dans nos têtes ces perceptions brutes dont il tient lieu. Mais nous le faisons à partir d'un matériau qui est le résultat d'une double abstraction et souvent d'une double substitution d'organes de perception. Nous sommes donc très éloignés de la perception brute de la réalité représentée. Beaucoup plus loin que nous ne le sommes avec le film. Cet éloignement est une des limites du texte. Il rend difficile la reconstitution exacte de la perception brute des choses. Ce même éloignement est aussi une des grandes qualités du texte car il nous donne une très grande liberté dans notre reconstitution de ce qu'aurait pu être la perception brute. Non seulement le texte nous donne-t-il une grande liberté dans cette reconstitution mais il oblige à l'exercer: on ne lit pas sans imaginer.

Le film ressemble beaucoup plus au rêve que le texte mais permet beaucoup moins que lui de rêver. Dit autrement, la morphologie du film - avec ses images et ses sons - est beaucoup plus près de celle du rêve que la morphologie du texte avec ses signes conventionnels sur du papier - et cela conduit à un effet paradoxal: le film qui ressemble au rêve ne le permet que très peu tandis que le texte qui ne lui ressemble pas le commande. Le film mime donc de beaucoup plus près la perception brute des choses que le texte et nous impose sa vision.

Le texte suscite aussi des sensations liées à tous les sens. De plus, il est à cheval entre les façons d'opérer du film et du rêve: celui qui lit reçoit ses balises de l'extérieur, comme avec le film, mais génère ses images de l'intérieur, comme le rêve. Le texte libère aussi le lecteur de son corps, comme le rêve et le film, et comme eux lui donne liberté totale de mouvement dans l'espace. Le texte apparaît ainsi une manifestation trompe-sens et trompegravité comme le film et le rêve.

Une distinction doit cependant être apportée. Dans le rêve et le texte, l'espace est illimité. Dans le texte il est perçu au second degré, dans le film et le rêve au premier. Dans le film il est bidimensionnel et cadré. Cette limite du cadre, qui peut sembler au premier abord être une entrave majeure à la capacité de représentation du film, est devenue au contraire, comme on le sait, un de ses principaux outils d'expression. 


\section{Liberté d'espaces et de temps}

Dans leur présentation, le film, le texte et le rêve partagent la même liberté totale de choix et d'agencement de leurs représentations de l'espace et du temps. Au premier degré pour le film et le rêve, au second pour le texte. Liberté de lieu. Liberté de point de vue dans le lieu (l'aspect trompe-gravité dont nous avons parlé plus haut). Liberté d'époque. Liberté aussi et surtout, nous semble-t-il, d'intensité de temps. En effet, lorsqu'on dit que le temps est dilaté, comprimé, accéléré, explosé par exemple, c'est surtout l'intensité de l'émotion du spectateur, du lecteur ou du rêveur qui se module ou qu'on module. Ce temps vécu nous semble le même dans les trois activités.

Nous avons donc plusieurs grandes caractéristiques communes à nos trois activités symboliques:

- déroulement hors du contrôle de ceux qui s'y adonnent;

- manifestation en trompe-sens et trompe-gravité;

- liberté d'espace et de temps;

- temps d'intensité variable.

Cette remarquable convergence des présentations en appelle de plus profondes.

\section{Pertinence, cohérence, satisfaction}

Rêve et émotions sont inextricablement liés. Rêve et rêveur ne font qu'un; émetteur et récepteur ne font qu'un; le rêveur est acteur à la fois de et dans son rêve. Acteur dans son rêve, signifiant par là qu'il peut $\mathrm{y}$ figurer de façon explicite en tant que personnage. Acteur de son rêve, parce que le rêve venant de lui, étant sa fabrication, ne peut présenter, au sens strict, autre chose que des facettes de lui sous le couvert de représentations diverses. Le rêve serait en effet, selon plusieurs ${ }^{1}$, la traduction en perceptions sensorielles d'une trame ou d'un nœud affectif du rêveur. Il en découle, par définition, que le rêve est toujours pertinent et cohérent même s'il laisse parfois perplexe. Le rêveur sait que le rêve le concerne. Le rêve n'est cependant pas obligatoirement satisfaisant, il agace parfois ou dérange.

Film et spectateur, texte et lecteur sont distincts l'un de l'autre. Émetteur et récepteur sont distincts; le spectateur ou le lecteur n'est (habituellement) ni acteur dans le film ou le texte. Le film ou le texte leur arrivant de l'extérieur ne sont pas toujours pertinents, ni cohérents ni satisfaisants. Nous proposons que la seule façon dont le film et le texte puissent être pertinents est qu'ils soient en résonance, faute de terminologie plus exacte, avec une trame ou un nœud affectif chez ceux qui les fréquentent. Cette résonance aurait lieu lorsque le film et le texte arri- 
veraient à se présenter comme une symbolisation adéquate et acceptable de la dite trame, ou nœud affectif.

Pour ce qui est de la cohérence du film et du texte, elle pourrait être vue comme le maintien, le transport de leur pertinence à travers toute la durée de l'œuvre. La recherche de la cohérence oblige à créer un mouvement, un renouvellement constant de la pertinence; renouvellement qui doit se faire - malgré ce que cela comporte de paradoxal — dans la continuité.

Pertinence et cohérence psychologiques donc, du film et du texte. Cela ne signifie pas que les personnages, les intrigues, etc. qu'on y retrouve doivent être réalistes et cohérents psychologiquement en tant que personnages, intrigues, etc.; cela ne signifie pas que tous les films et les textes doivent se tenir dans leurs personnages et leurs récits comme se tiennent les drames psychologiques, par exemple. Cohérence psychologique signifie que ce qui se passe dans le film et le texte doit être cohérent psychologiquement, symboliquement, pour le spectateur ou le lecteur. Nous entendons par là que le- film et le texte doivent correspondre à une certaine trame ou à un certain nœud affectif de leur public; doivent correspondre à leurs désirs, à leurs peurs, à leurs fantasmes, à leur dynamique affective, et cela de façon consciente ou pas. Une des stratégies privilégiées pour atteindre cette cohérence et pour la véhiculer est de l'incarner dans la psychologie des personnages et des intrigues. Mais cette façon de procéder n'est pas nécessairement un prérequis à la cohérence psychologique du film et du texte. Cette dernière est atteinte lorsque le film ou le texte réussissent à se faire les miroirs de l'âme de leur public, peu importe les moyens utilisés.

Selon cette vision, le film et le texte opéreraient de trois façons principales pour réussir leur jonction avec leur public:

- Ils se feraient miroir symbolique de la trame affective du public. Cet effet de miroir serait perçu de façon soit consciente ou inconsciente par lui.

Dans le cas où l'effet serait conscient, on peut se demander à quoi servirait la démarche symbolique. Elle servirait probablement et surtout lorsque la conscience de cette trame ou son énonciation brute doit rester cachée, ou n'est pas permise; dans des films qui, par la bande, sont des critiques sociales dans des régimes répressifs, par exemple.

Conscient ou inconscient, dans les deux cas, l'effet serait de soulever et de canaliser les émotions. Et l'intensité de ces émotions serait probablement proportionnelle à la profondeur de la trame visée ou rejointe, au poids du non-dit dans un cas, ou du non consciemment perçu dans l'autre. 
- La deuxième façon dont le film ou le texte opéreraient pour établir leur jonction avec le public serait en jouant le rôle de révélateur symbolique d'une trame ou d'un nœud affectif jusque-là caché, sinon aux yeux du moins à la conscience du spectateur.

- La troisième stratégie serait de se présenter en tant que réponse symbolique, résolution symbolique d'une trame ou d'un nœud affectif chez le spectateur.

Dans la réalité, ce serait par une combinaison de ces trois stratégies - miroir, révélateur, solution symboliques - que le film et le texte tenteraient de se marier à la trame affective de leur public.

Donc, si le rêve est le signe des émotions du rêveur, le texte et le film, eux, pourraient être des propositions symboliques faites aux gens d'une trame ou d'un nœud affectif chez eux: des rêves, ou des quasi-rêves.

Le rêve n'a pas à être satisfaisant pour le rêveur. Le film et le texte, oui. En effet, c'est contre la promesse d'en être satisfaits que les gens acceptent de se mettre en disponibilité face au film et au texte. Dans cette transaction, chacun met du sien: les gens apportent leur participation; le film et le texte, leur présentation.

\section{Les apports du public}

Le tout premier apport des gens vient d'une attitude bienveillante à l'égard du film et du texte: ils veulent réussir leur transaction, ils désirent en être satisfaits.

Le deuxième apport des gens vient du fait que l'être humain est un animal qui a soif de sens, de pertinence et de cohérence, et qu'il fera beaucoup pour en insuffler à quoi que ce ce soit qu'on lui présente, surtout s'il s'attend à en être satisfait. Les gens participent activement à la création du sens, de la cohérence et de la pertinence du film et du texte. Le film et le texte cherchent à marier leurs symboles à la trame affective de leur public; le public cherche à marier sa trame affective à une représentation symbolique pertinente, cohérente et satisfaisante, quitte à s'aider un peu en cours de route si nécessaire.

Certaines participations sont bien connues: les gens établissent la continuité entre les images du film, entre les phrases du texte; ils comblent les espaces, les temps et le récit entre les plans, entre les scènes, entre les séquences du film, entre les phrases, entre les «images», entre les paragraphes du texte.

Le troisième apport des gens à la transaction avec le film et le texte vient de la suspension de leur incrédulité. Ce que certains 
appellent la crédulité volontaire. Les gens acceptent en effet, jusqu'à un certain point, de ne pas juger rationnellement ce qu'on leur présente. Ils adhèrent à l'œuvre malgré les entorses possibles à la logique ou à la réalité physique. Cette suspension de l'incrédulité est une démarche vraiment extraordinaire lorsqu'on l'examine par rapport à l'ordre ordinaire des choses; tout alors nous dit que nous ne devrions pas y adhérer. Le fait que nous le fassions nous apparaît comme une confirmation éclatante du fait que la réalité première du film et du texte est justement ailleurs que dans cet ordre ordinaire des choses. $\mathrm{Si}$ l'on accepte que la réalité première du film et du texte est dans leur fondement symbolique et que leur pertinence et leur cohérence sont psychologiques, la crédulité volontaire devient alors une chose qui va de soi. C'est effectivement le cas dans notre fréquentation ordinaire de l'audiovisuel et du texte. Ce qui nous concerne ce n'est pas d'abord la rationalité du film ou du texte; ce qui nous intéresse c'est le fait qu'ils nous rejoignent au niveau de nos désirs, de nos peurs, de nos fantasmes, de notre émerveillement, quelles que soient les entorses aux lois de la physique ou le manque de réalisme de la représentation.

La suspension de l'incrédulité n'est ni absolue ni donnée au départ. Elle doit être acquise et maintenue. Elle est volontaire et liée à certaines conditions. Les gens affichent un niveau de tolérance variable en ce qui la touche selon les genres, selon les conventions annoncées par le film ou le texte, connues et respectées de part et d'autre. La suspension de l'incrédulité est une des composantes essentielles de la transaction film-spectateur, ou texte-lecteur.

Nous avons donc trois grandes participations des gens à leurs transactions avec le film et le texte:

- une bienveillance initiale;

- un apport actif à la formation du récit et du sens;

- une suspension de leur incrédulité.

\section{Les apports du film et du texte}

Tout d'abord, le film ou le texte qui réussit sa transaction ne laisse jamais perplexe. Il caractérise toujours bien ses représentations; il en établit clairement les Qui, Que, Quoi, Où, Quand, Comment et Pourquoi. Il permet une lecture claire, au premier degré, de ses éléments, de leur état et de leur articulation les uns par rapport aux autres. C'est une des conditions de base, insuffisante à elle seule, mais absolument nécessaire à la mise en disponibilité volontaire des gens et à leur crédulité volontaire. Ce n'est qu'une fois situés qu'ils seront en mesure de sentir si ce qui 
leur est proposé les concerne. Obligation de clarté initiale donc, qui se répercute et se transporte d'un bout à l'autre de l'œuvre.

Le film et le texte sont distincts de ceux qui les fréquentent alors que rêve et rêveur ne font qu'un. Rêve et émotions sont inextricablement liés tandis que le film et le texte sont d'un côté et leur public de l'autre. Le film et le texte essaient de recréer cette unité rêveur-rêve (l'unité émotion-représentation... ), et mettent en œuvre plusieurs stratégies à cette fin. D'abord, pour éviter d'avoir constamment à remorquer émotivement les gens, le film et le texte tentent de les projeter vers l'avant. Ils tentent de leur faire devancer émotivement le déroulement de l'œuvre. Non seulement cela évite-t-il d'avoir à les remorquer, mais cela recrée aussi chez eux les conditions du rêve, à savoir que les représentations semblent alors répondre à la trame affective, semblent procéder d'elle, que l'une et l'autre semblent alors s'appartenir comme c'est le cas dans le rêve. L'outil de cette projection émotive des gens vers l'avant est la construction dramatique au sens large. Elle est au cœur du fonctionnement du film et du texte. Et cela, croyons-nous, même chez ceux dont la vocation n'est pas «dramatique» ou fictionnelle.

Le film, qui mime de très près le rêve, utilise un instrument additionnel qui lui est spécifique pour recréer l'unité rêve-rêveur. Cet instrument est la «musique de film». Par musique de film, nous entendons la musique bien sûr, mais aussi tout l'arrière-plan sonore du film dont l'objet est de servir l'intention dramatique du moment, c'est-à-dire de servir ce que l'on veut alors faire sentir au spectateur. La présentation du film incorpore une trame de représentations qui se veut explicitement être celle des émotions du spectateur vis à vis des autres représentations de l'œuvre. Ce rapprochement mime de près l'unité rêverêveur.

\section{Le cas du scénario}

Le texte cherche, tout comme le film, à recréer l'unité rêverêveur. Il n'essaie cependant pas, comme le film, de mimer le rêve. Il y va directement, il balise une chose qui est $a ̀$ rêver par le lecteur. C'est sa force. Mais à partir des balises du texte, chacun fait son propre rêve, et cela nous pose une des grandes problématiques du scénario: comment faire rêver le même film à tous les lecteurs; comment faire rêver au film qu'on veut à l'écran et à celui-là seulement? Le scénario s'y efforce en adoptant deux grandes stratégies: le mimétisme de l'agencement des grands éléments du film et l'écriture descriptive.

Le scénario reproduit par sa mise en page une grande partie de l'agencement des éléments du film: 
- il sépare graphiquement le son et l'image comme ils sont séparés dans le film. Au premier regard, on voit la pondération dialogues-images; on voit le rythme des dialogues.

- le scénario sépare graphiquement les lieux les uns des autres, en faisant de chaque changement de lieu un changement de scène.

- le scénario adopte aussi un niveau de définition constant d'écriture. Il illustre par le fait même la durée relative de chaque événement, scène ou séquence du film.

- par les appels des scènes, le scénario montre la pondération jour et nuit, intérieur et extérieur du film et aussi la récurrence de chacun des lieux à travers le film.

- par le découpage implicite inscrit dans ses descriptions, le scénario mime le cadrage, la valeur des plans, la profondeur de champ et le montage.

Sauf dans les dialogues, le scénario adopte une nette dominante d'écriture descriptive plutôt qu'évocative; il cherche à utiliser le moins possible de mots qui «font image», et le plus possible à décrire les images et les sons avec des mots «plats», lesquels sons et images trouvent leur résonance une fois décrits. Le niveau de définition de l'écriture descriptive du scénario suit celui du film allant jusqu'au plan pour ce qui est de mimer sa forme et jusqu'au moment dramatique pour ce qui est du fond.

Ce sont là les principales spécificités de présentation du scénario visant à réduire la marge d'interprétation qu'on peut en faire. Ces stratégies ne sont que partiellement efficaces. À partir des balises du scénario, le réalisateur fera son propre rêve et c'est ce rêve qu'on verra à l'écran. Cela est confirmé par la pratique des notes de réalisation qui vient réduire la marge d'incertitude liée à cette dérive obligatoire. On demande souvent en effet au réalisateur de spécifier sa vision du film à partir du scénario avant de le produire. Ainsi, dans la production d'un film (lorsqu'il y a scénario) et après le scénario, tout le monde travaille en interprétation. C'est une évidence que vient confirmer la pratique des notes de réalisation. Évidence qui soulève la rogne d'une certaine école de réalisateurs qui, malgré le quotidien de leur métier et la longueur des génériques, s'entêtent à croire que le cinéma est un art solo.

Rappelons à cet égard qu'à l'écriture du scénario, le film n'existe pas. L'obligation que se donne le scénario de mimer le 
film entraîne donc d'abord celle de l'inventer, au complet (univers, personnages, action, récit, film) ou bien à partir d'une œuvre préexistante, ce qui, dans la pratique, est une tâche tout aussi formidable. Rappelons aussi qu'après l'écriture du scénario qui l'invente, le film n'existe toujours pas. Dans leur transaction avec les gens, le film et le texte apportent donc la clarté et la construction dramatique; le film apporte en plus la musique de film tandis que le scénario, là où il existe, apporte le mimétisme du film, l'écriture descriptive et (une grande partie de) la création.

Pour résumer et conclure cette réflexion sur le film, le texte et le rêve en tant qu'objets et activités symboliques, nous dirons qu'elle a déjà modifié la perception que nous avons de notre travail de scénariste, qu'elle a subtilement mais de façon certaine modifié la façon dont nous l'entreprenons et qu'elle a bouleversé la façon dont nous l'enseignons. Finalement, nous ne résisterons pas à la tentation d'aligner les trois énoncés suivants, tout en en reconnaissant déjà la circularité, les limites et les exagérations:

- Le rêve est un film qui surgit dans la tête du rêveur;

- Le film est un rêve imposé au spectateur;

- Le texte est un rêve balisé pour le lecteur.

Université de Montréal

\section{NOTE}

1 Voir à ce sujet Jonathan Winson, «The Meaning of Dreams», Scientific American, vol. 263, no 5 (nov. 1990). 